\title{
Commentary \\ Recent translational research: Oncogene discovery by insertional mutagenesis gets a new boost
}

\author{
John Hilkens
}

Division of Tumor Biology, The Netherlands Cancer Institute, Amsterdam, The Netherlands

Corresponding author: John Hilkens, j.hilkens@nki.nl

Published: 16 January 2006

This article is online at http://breast-cancer-research.com/content/8/1/102

(c) 2006 BioMed Central Ltd

Breast Cancer Research 2006, 8:102 (doi:10.1186/bcr1376)

\begin{abstract}
Knowledge of the genes and genetic pathways involved in oncogenesis is essential if we are to identify novel targets for cancer therapy. Insertional mutagenesis in mouse models is among the most efficient tools to detect novel cancer genes. Retrovirusmediated insertional mutagenesis received a tremendous boost by the availability of the mouse genome sequence and new PCR methods. Application of such advances were limited to lymphomagenesis but are now also being applied to mammary tumourigenesis. Novel transposons that allow insertional mutagenesis studies to be conducted in tumors of any mouse tissue may give cancer gene discovery a further boost.
\end{abstract}

\section{Introduction}

Oncogenic transformation of a normal cell is a multistep process that requires activation or inactivation, usually by mutagenic events, of several key genes controlling various essential cellular pathways that are involved in growth and development. An understanding of which pathways and genes are involved is essential to the development of novel therapeutic strategies. Although a large number of genes involved in breast and other cancers has been discovered, the picture is far from complete. Retroviral insertional mutagenesis (IM) in mouse models is among the most efficient tools to uncover cancer genes. The most important advantage of IM over chemical and radiation induced mutagenesis is that the mutagen is also a molecular tag, facilitating the identification of the mutated locus. Because proviral integration is essentially random with some bias for transcriptionally active domains, retroviral insertions in the same genomic locus in multiple independent tumours mark a so-called common insertion site (CIS), which is likely to contain a gene involved in tumourigenesis.

IM was first described in 1981 by Hayward and coworkers [1] when those investigators, in an effort to elucidate the mechanism by which avian leukosis virus induces lymphomas, discovered that the virus targeted the cellular Myc gene. Shortly thereafter, Nusse and Varmus [2] identified Wnt1 as a frequent target of mouse mammary tumour virus (MMTV) using this approach. IM mediated by retroviruses is based on the integration of a DNA copy of retroviral RNA as a provirus into the cellular genome as part of the life cycle of the virus. The regulatory elements in the proviral long terminal repeats can then transcriptionally activate adjacent cellular genes. Proviral insertions that truncate cellular genes giving rise to dysfunctional or constitutively active proteins are also frequently found; for an extensive overview of retroviral IM, see the reports by Jonkers and Berns [3] and Mikkers and Berns [4].

Although these landmark studies were followed by several others, the number of oncogenes discovered in this way remained rather limited. This was mainly due to the rather laborious techniques, including Southern blotting, extensive cloning of DNA fragments and 'chromosome walking', needed to identify the affected gene. The availability of novel PCR technologies such as the splinkerette [5] and inverse PCR [6], but in particular the completion of the draft sequence of the mouse genome, permitted high-throughput screening strategies and caused a revival of the IM approach to gene discovery. Hundreds of novel candidate oncogenes involved in lymphomagenesis have recently been reported using Moloney murine leukaemia virus (MoMuLV) [5,7-10]. Despite these technological advances, no large-scale screening for CISs in other neoplasias was conducted until very recently. Here I review some of the recent papers that used new opportunities for gene discovery in brain and mammary tumourigenesis $[8,11,12]$. In addition, I discuss transposon-mediated IM in mice, which has now achieved a degree of maturity and in theory allows high-throughput cancer gene discovery studies in any tissue.

\section{Insertional mutagenesis in mouse mammary tumours}

Thus far, no more than 12 genes with proven oncogenicity have been found by MMTV tagging in mouse mammary

$\mathrm{CIS}=$ common insertion site; IM = insertional mutagenesis; MMTV = mouse mammary tumour virus; MoMuLV = Moloney murine leukaemia virus; $\mathrm{PB}=$ PiggyBac transposon; $\mathrm{PCR}=$ polymerase chain reaction; PDGF = platelet-derived growth factor; $\mathrm{SB}=$ Sleeping Beauty transposon. 
Table 1

\begin{tabular}{|c|c|c|c|c|}
\hline Gene & Gene product & $\begin{array}{l}\text { Confirmed } \\
\text { oncogenicity }\end{array}$ & $\begin{array}{l}\text { Evidence for involvement } \\
\text { in human breast cancer }\end{array}$ & Reference \\
\hline Fgf3/int2 & Fibroblast growth factor & Yes & No & {$[22]$} \\
\hline Fgf4 & Fibroblast growth factor & Yes & No & [23] \\
\hline Fgf8 & Fibroblast growth factor & Yes & Yes & [24] \\
\hline Fgf10 & Fibroblast growth factor & Yes & Yes & [11] \\
\hline Int-5/Int-H & Aromatase & Yes & Yes & {$[25]$} \\
\hline Int6/Eif3e & Translation initiation factor 3 subunit & Yes & No & {$[26]$} \\
\hline $\operatorname{lnt7}$ & Thrombospondin domain containing gene & No & No & [12] \\
\hline Int-41 & Unknown & No & No & {$[27]$} \\
\hline Map3k8/Tp/2 & Mitogen-activated protein kinase & Yes & Yes & {$[28]$} \\
\hline Notch1 & Receptor/transcription cofactor precursor & Yes & Yes & [29] \\
\hline Notch4/Int3 & Receptor/transcription cofactor precursor & Yes & No & [30] \\
\hline Wnt/int1 & Morphogen & Yes & No & [2] \\
\hline Wnt3 & Morphogen & Yes & No & [31] \\
\hline Wnt10b & Morphogen & Yes & Yes & [32] \\
\hline
\end{tabular}

MMTV, mouse mammary tumour virus.

tumours (Table 1). One of these (Int5) may only be involved in hormone-dependent growth, whereas the oncogenic potential of two genes (Int41 and the novel Int7 gene [12]) remains to be established. Although not all of these genes are involved in human breast cancer, most of the pathways in which they act have been implicated in human disease. Remarkably, the list of confirmed oncogenes is mainly composed of Wnt, Fgf and Notch genes, which are involved in development of the embryonic mammary gland. Int7 belongs to a novel class of oncogenes [12]. Although its oncogenicity is yet to be established, its discovery shows that MMTV-mediated IM still has the potential to identify new classes of oncogenes in mammary tumourigenesis. Also, the sky rocketing number of candidate oncogenes found in the high-throughput MoMuLV mediated IM studies in lymphomagenesis indicates that MMTV-mediated gene tagging in mammary tumourigenesis needs further exploration. An obvious question arising is whether proviral MoMuLV and MMTV may tag the same genes. Comparison of the loci tagged by MMTV in mammary tumours identified in our own recent semi-high-throughput study (Theodorou V, Boer M, Kimm M, Theelen W, Jonkers J, Hilkens J, unpublished data) with the loci tagged by MoMuLV in murine lymphomas deposited in the mouse Retrovirus Tagged Cancer Gene Database [13] suggests that the overlap is small. This indicates that the cancer-associated signal transduction pathways in mammary tumourigenesis and lymphomagenesis are at least partly different and warrants further high-throughput IM studies.

\section{New technological developments Insertional mutagenesis with modified retroviruses}

Collaborating oncogenes (i.e. genes that act together in tumourigenesis) can also be identified by IM because they are frequently coactivated in the same tumours. IM in oncogene-expressing transgenic mice is frequently used to identify oncogene collaboration more directly. This method was used to show, for example, that Wnt and Fgf genes collaborate in mammary tumourigenesis [14]. Recently, Johansson and coworkers [8] used another IM approach to seek collaborating genes. Based on the notion that autocrine stimulation of the PDGF receptors is a frequent event in glioblastoma development, they administered to mice an intracerebral injection of recombinant MoMuLV encoding platelet-derived growth factor (PDGF)- $\beta$ (together with a wildtype MoMuLV helper virus). The recombinant virus not only predisposed these mice to gliomas, but MoMuLV also efficiently tagged oncogenes in the brain, potentially collaborating with PDGF- $\beta$. This experiment also shows that nonhaematopoietic cells can be predisposed in such a way that these cells accumulate sufficient insertions to develop into tumours before haematopoietic cells, which are the prime targets of MoMuLV, can do so. However, local virus injection may also have contributed to the absence of lymphomas in these animals.

The authors identified 66 loci that harbour potential brain tumour-associated oncogenes that collaborate with PDGF- $\beta$. In a subsequent report [15], it was shown that most of the common retroviral insertions indeed affected the predicted genes - evidence that recent high-throughput studies often lack. Some of these candidate oncogenes were previously not known to be involved in (brain) tumourigenesis.

\section{Transposon-mediated cancer gene tagging}

Until recently, retrovirally induced IM was not applied in solid tumours with the exception of mouse mammary tumours and 
the brain tumours discussed above because of tissue tropism of the retroviruses. Transposon-mediated IM may be more widely applicable and is highly efficient, as has been shown in Caenorhabtidis elegans and Drosophila [16,17]. DNA transposons are discrete DNA elements that are able to translocate within the cellular genome by excision and reinsertion mediated by a transposase, which can be encoded by the transposable DNA element or can be located elsewhere in the genome. No active DNA transposons have been identified in mammalian genomes. However, recently no fewer than three papers described artificial transposable elements designed for IM in the mouse. Dupuy [18] and Collier [19] and their coworkers used an adapted Sleeping Beauty (SB) transposon that was originally resurrected from evolutionary inactive elements in fish DNA, whereas Ding and colleagues [20] adapted PiggyBac (PB) derived from a DNA transposable element in a cabbage moth. Both are binary systems (i.e. the transposase and transposon are independently transmitted and can be maintained in different animals that can be interbred to mobilize the transposon). PB was shown to efficiently integrate and transpose randomly not only in cell lines but also in the mouse germ line. This transposon may thus be particularly useful for germ line IM. However, the low number of transpositions per generation or cell division may limit the use of PB for cancer gene discovery in its present form.

Dupuy [18] and Collier [19] and their coworkers have adapted the SB system more specifically for IM in somatic cells by providing the transposon with viral promoter and enhancer elements for gene activation, and splice donor, acceptor and polyadenylation sequences for induction of intragenic insertional mutations by gene trapping. SB efficiently mobilizes in somatic cells and reinserts in or close to genes, thereby modifying gene expression and/or interrupting coding sequences by similar mechanisms as employed by retroviruses. However, DNA transposons hop through the genome by a cut and paste mechanism and proliferate very slowly, which is inhibitory for efficient somatic mutagenesis. Dupuy [18] and Collier [19] and their coworkers solved this problem with SB to some extent by introducing large concatamers containing up to 300 copies of the transposon into the mouse genome. A shortcoming of the SB system used by Collier and coworkers [19] is its tendency to transpose to locations close to the excision site. This means that even after several rounds of transposition the majority of the transposons still tend to land on the same chromosome. The transposon density on the originating chromosome can be so high that the significance of the less frequently tagged loci may be doubted. Although mobilization of the Dupuy variant was more efficient, allowing the transposons to spread wider and to different chromosomes, this system suffered from a high incidence of embryonic lethality and a strong predispostion to haematopoietic tumours. Both issues may be resolved by the use of tissue-specific and/or inducible SBbased mutagenesis systems, in which tissue specificity and/or timing of transposase activity can be controlled.

\section{Conclusion}

Discovery of new genes with a causal role in cancer and delineation of the pathways through which these genes act form the basis for designing novel cancer treatments. Retroviral IM has already proven to be invaluable for this purpose and transposon-mediated IM will broaden further its application to malignancies other than lymphomas and mammary tumours. Because lymphoma genes tagged by transposons only partly overlap with genes targeted by retroviruses, both technologies may complement each other.

Exhaustive screening for IM screens will yield many genes with such a low contribution to oncogenesis that therapeutic targeting of these genes or gene products is ineffective. This may be particularly true for infrequently hit genes. Moreover, the availability of the Retrovirus Tagged Cancer Gene Database [13] in principle allows different studies to be combined, and it may be tempting to conclude that loci that are sporadically hit in separate studies are common, without consideration of statistics. However, the genomic window in which the insertions must be present for a statistically meaningful $\mathrm{CIS}$ will become very small as the number of analyzed insertions increases [5]. The size of such windows may even be overestimated, because both proviruses and transposons tend to land preferentially in transcriptionally active regions of the genome [21]. Furthermore, the affected gene may not always simply be the adjacent gene. This means that candidate cancer genes identified from IM screens should ultimately be validated in an oncogenicity study.

Another point to consider is that cancer genes may affect different tissues when they are mutated in mice and in humans; therefore, conclusions of experiments obtained with murine cancer genes should be carefully assessed before applying them to human cancer. Searching the available microarray expression data for over-expression of the studied mouse gene in human cancer may already provide a quick indication of the relevance of this gene for human cancer.

Extrapolating from the number of genes found by $\mathrm{IM}$ in lymphomas, we may expect a large number of novel candidate oncogenes to be discovered by IM in other tissues. In mammary carcinogenesis the novel genes will come from MMTV-mediated IM, and the list will probably be completed by transposon tagging. In other tumours the full benefit of transposon-mediated IM will be obtained. The next challenge will be to prove the true contributions of all these genes to oncogenesis, to find the pathways in which they act, and to exploit this knowledge for therapy.

\section{Competing interests}

The author(s) declare that they have no competing interests.

\section{Acknowledgement}

I am very grateful to Dr J Jonkers, Division of Molecular Biology, The Netherlands Cancer Institute for his comments and critical reading of the manuscript. 


\section{References}

1. Hayward WS, Neel BG, Astrin SM: Activation of a cellular onc gene by promoter insertion in ALV-induced lymphoid leukosis. Nature 1981, 290:475-480.

2. Nusse R, Varmus HE: Many tumors induced by the mouse mammary tumor virus contain a provirus integrated in the same region of the host genome. Cell 1982, 31:99-109.

3. Jonkers J, Berns A: Retroviral insertional mutagenesis as a strategy to identify cancer genes. Biochim Biophys Acta 1996, 1287:29-57.

4. Mikkers $\mathrm{H}$, Berns $\mathrm{A}$ : Retroviral insertional mutagenesis: tagging cancer pathways. Adv Cancer Res 2003, 88:53-99.

5. Mikkers H, Allen J, Knipscheer P, Romeijn L, Hart A, Vink E, Berns A, Romeyn L: High-throughput retroviral tagging to identify components of specific signaling pathways in cancer. Nat Genet 2002, 32:153-159.

6. Li J, Shen H, Himmel KL, Dupuy AJ, Largaespada DA, Nakamura T, Shaughnessy JDJ, Jenkins NA, Copeland NG: Leukaemia disease genes: large-scale cloning and pathway predictions. Nat Genet 1999, 23:348-353.

7. Suzuki T, Shen H, Akagi K, Morse HC, Malley JD, Naiman DQ, Jenkins NA, Copeland NG: New genes involved in cancer identified by retroviral tagging. Nat Genet 2002, 32:166-174.

8. Johansson FK, Brodd J, Eklof C, Ferletta M, Hesselager G, Tiger CF, Uhrbom L, Westermark B: Identification of candidate cancer-causing genes in mouse brain tumors by retroviral tagging. Proc Natl Acad Sci USA 2004, 101: 11334-11337.

9. Lund $\mathrm{AH}$, Turner $\mathrm{G}$, Trubetskoy A, Verhoeven $\mathrm{E}$, Wientjens $\mathrm{E}$, Hulsman D, Russell R, DePinho RA, Lenz J, van Lohuizen M: Genome-wide retroviral insertional tagging of genes involved in cancer in Cdkn2a-deficient mice. Nat Genet 2002, 32:160165.

10. Shin MS, Fredrickson TN, Hartley JW, Suzuki T, Agaki K, Morse $\mathrm{HC}$, III: High-throughput retroviral tagging for identification of genes involved in initiation and progression of mouse splenic marginal zone lymphomas. Cancer Res 2004, 64:4419-4427.

11. Theodorou V, Boer M, Weigelt B, Jonkers J, Van der Valk M, Hilkens J: Fgf10 is an oncogene activated by MMTV insertional mutagenesis in mouse mammary tumors and overexpressed in a subset of human breast carcinomas. Oncogene 2004, 23:6047-6055.

12. Lowther W, Wiley K, Smith GH, Callahan R: A new common integration site, Int7, for the mouse mammary tumor virus in mouse mammary tumors identifies a gene whose product has furin-like and thrombospondin-like sequences. $J$ Virol 2005, 79:10093-10096.

13. Mouse Retrovirus Tagged Cancer Gene Database (RTCGD) [http://RTCGD.ncifcrf.gov]

14. Shackleford GM, MacArthur CA, Kwan HC, Varmus HE: Mouse mammary tumor virus infection accelerates mammary carcinogenesis in Wnt-1 transgenic mice by insertional activation of int-2/Fgf-3 and hst/Fgf-4. Proc Natl Acad Sci USA 1993, 90:740-744.

15. Johansson FK, Goransson H, Westermark B: Expression analysis of genes involved in brain tumor progression driven by retroviral insertional mutagenesis in mice. Oncogene 2005, 24:3896-3905.

16. Zwaal RR, Broeks A, van Meurs J, Groenen JT, Plasterk RH: Target-selected gene inactivation in Caenorhabditis elegans by using a frozen transposon insertion mutant bank. Proc Natl Acad Sci USA 1993, 90:7431-7435.

17. Spradling AC, Stern DM, Kiss I, Roote J, Laverty T, Rubin GM: Gene disruptions using $P$ transposable elements: an integral component of the Drosophila genome project. Proc Natl Acad Sci USA 1995, 92:10824-10830.

18. Dupuy AJ, Akagi K, Largaespada DA, Copeland NG, Jenkins NA: Mammalian mutagenesis using a highly mobile somatic Sleeping Beauty transposon system. Nature 2005, 436:221226.

19. Collier LS, Carlson CM, Ravimohan S, Dupuy AJ, Largaespada DA: Cancer gene discovery in solid tumours using transposon-based somatic mutagenesis in the mouse. Nature 2005, 436:272-276

20. Ding S, Wu X, Li G, Han M, Zhuang Y, Xu T: Efficient transposition of the piggyBac (PB) transposon in mammalian cells and mice. Cell 2005, 122:473-483.
21. Mitchell RS, Beitzel BF, Schroder AR, Shinn P, Chen H, Berry CC, Ecker JR, Bushman FD: Retroviral DNA integration: ASLV, HIV, and MLV show distinct target site preferences. PLoS Biol 2004, 2:E234.

22. Peters G, Brookes S, Smith R, Dickson C: Tumorigenesis by mouse mammary tumor virus: evidence for a common region for provirus integration in mammary tumors. Cell 1983, 33:369-377.

23. Peters G, Brookes S, Smith R, Placzek M, Dickson C: The mouse homolog of the hst/k-FGF gene is adjacent to int-2 and is activated by proviral insertion in some virally induced mammary tumors. Proc Natl Acad Sci USA 1989, 86:56785682.

24. MacArthur CA, Shankar DB, Shackleford GM: Fgf-8, activated by proviral insertion, cooperates with the Wnt- 1 transgene in murine mammary tumorigenesis. J Virol 1995, 69:2501-2507.

25. Gray DA, McGrath CM, Jones RF, Morris VL: A common mouse mammary tumor virus integration site in chemically induced precancerous mammary hyperplasias. Virology 1986, 148:360368.

26. Marchetti A, Buttitta F, Miyazaki S, Gallahan D, Smith GH, Callahan R: Int-6, a highly conserved, widely expressed gene, is mutated by mouse mammary tumor virus in mammary preneoplasia. J Virol 1995, 69:1932-1938.

27. Garcia M, Wellinger $R$, Vessaz A, Diggelmann $H$ : A new site of integration for mouse mammary tumor virus proviral DNA common to $B A L B / c f(C 3 H)$ mammary and kidney adenocarcinomas. EMBO J 1986, 5:127-134.

28. Erny KM, Peli J, Lambert JF, Muller V, Diggelmann H: Involvement of the Tpl-2/cot oncogene in MMTV tumorigenesis. Oncogene 1996, 13:2015-2020.

29. Girard L, Hanna Z, Beaulieu N, Hoemann CD, Simard C, Kozak $\mathrm{CA}$, Jolicoeur $\mathrm{P}$ : Frequent provirus insertional mutagenesis of Notch1 in thymomas of MMTVD/myc transgenic mice suggests a collaboration of c-myc and Notch1 for oncogenesis. Genes Dev 1996, 10:1930-1944.

30. Gallahan D, Callahan R: Mammary tumorigenesis in feral mice: identification of a new int locus in mouse mammary tumor virus (Czech II)-induced mammary tumors. J Virol 1987, 61:66-74.

31. Roelink H, Wagenaar E, Lopes dS, Nusse R: Wnt-3, a gene activated by proviral insertion in mouse mammary tumors, is homologous to int-1/Wnt-1 and is normally expressed in mouse embryos and adult brain. Proc Natl Acad Sci USA 1990, 87:4519-4523.

32. Lee FS, Lane TF, Kuo A, Shackleford GM, Leder P: Insertional mutagenesis identifies a member of the Wnt gene family as a candidate oncogene in the mammary epithelium of int-2/Fgf3 transgenic mice. Proc Natl Acad Sci USA 1995, 92:22682272 